\title{
Vorwort zur achten Auflage
}

Für die achte Auflage habe ich den ganzen Inhalt des Buches wieder einmal überarbeitet, und bei dieser Gelegenheit auch meinem Beweise des zweiten Hauptsatzes ( $\$ 118 \mathrm{ff}$.) insofern eine andere Fassung gegeben, als ich ihn nicht, wie bisher, zunächst auf ideale Gase, sondern gleich von vornherein auf beliebige Körper erstreckte, ebenso wie das C. Carathtodory bei seiner sorgfältigen axiomatischen Begründung des zweiten Hauptsatzes im 67. Band der Mathematischen Annalen, S. 355, vom Jahre 1909, durchgeführt hat. Von der Benutzung des Carathíodoryschen Ausgangsprinzips glaubte ich allerdings absehen zu sollen, wegen gewisser Bedenken, die ich an andrer Stelle, in den Sitzungsberichten der PreuBischen Akademie der Wissenschaften vom Jahre 1926, S. 453, auseinandergesetzt habe.

Eine wesentliche Verbesserung konnte auch die Darstellung der Thermodynamik starkdissoziierter Elektrolyte ( $\$ 273$ ) erfahren, da die in der Zwischenzeit erschienenen Arbeiten von P. DEBYE und E. Hückes durch ibre tiefere Erfassung des Problems einen bedeutenden Schritt über die von Gноsн erzielten Resultate hinausgeführt haben.

Endlich erwähne ich hier noch als besonders bemerkenswert die Tatsache, daB in neuester Zeit durch Messungen von F. Srmon die Nullpunktsentropie einer Lösung (§ 291) zum erstenmal nicht nur wohl einwandfrei nachgewiesen, sondern auch in merklicher Übereinstimmung mit der Theorie gefunden worden ist.

Berlin-Grunewald, im Januar 1927 